\title{
Combining Adoptive Cell Therapy with Cytomegalovirus-Based Vaccine Is Protective against Solid Skin Tumors
}

\author{
Jeremy M. Grenier ${ }^{1}$, Stephen T. Yeung ${ }^{1 \dagger}$, Zhijuan Qiu', Evan R. Jellison ${ }^{1}$ \\ and Kamal M. Khanna ${ }^{1 *}$ \\ ${ }^{1}$ Department of Immunology, University of Connecticut Health, Farmington, CT, United States, ${ }^{2}$ Department of Molecular \\ Genetics and Microbiology, Center for Infectious Diseases, Stony Brook University, Stony Brook, New York, NY, \\ United States
}

\section{OPEN ACCESS}

Edited by: Nurit Hollander,

Tel Aviv University, Israel

Reviewed by: Carlos Alfaro,

Universidad de Navarra, Spain Andrew Zloza,

Rutgers Cancer Institute of New Jersey, United States

*Correspondence:

Kamal M. Khanna kamal.khanna@nyumc.org

${ }^{\dagger}$ Present address:

Stephen T. Yeung and Kamal M. Khanna, Department of Microbiology,

Perlmutter Cancer Center, New York University School of Medicine, New York, NY, United States

Specialty section: This article was submitted to Cancer Immunity and Immunotherapy,

a section of the journal

Frontiers in Immunology

Received: 07 November 2017 Accepted: 22 December 2017 Published: 16 January 18

Citation:

Grenier JM, Yeung ST, Qiu Z, Jellison ER and Khanna KM (2018) Combining Adoptive Cell Therapy with Cytomegalovirus-Based Vaccine

Is Protective against Solid Skin Tumors.

Front. Immunol. 8:1993 doi: 10.3389/fimmu.2017.01993
Despite many years of research, cancer vaccines have largely been ineffective in the treatment of established cancers. Many barriers to immune-mediated destruction of malignant cells exist, and these likely limit the efficacy of cancer vaccines. In this study, we sought to enhance the efficacy of a cytomegalovirus (CMV)-based vaccine targeting melanoma by combining vaccination with other forms of immunotherapy. Adoptive cell therapy in humans and in animal models has been shown to be effective for tumor regression. Thus, in this study, we assessed whether CMV-based vaccines in combination with adoptively transferred antitumor $\mathrm{T}$ cells could provide greater antitumor protection than either therapy alone. Our results show that adoptive cell therapy greatly enhanced the antitumor effects of CMV-based vaccines targeting the foreign model antigen, OVA, or the melanoma differentiation antigen, gp100. Combination adoptive cell therapy and vaccination induced the upregulation of the inhibitory ligands, PD-L1, and Qa-1 ${ }^{\mathrm{b}}$, on B16 tumor cells. This expression paralleled the infiltration of tumors by vaccine-stimulated $T$ cells which also expressed high levels of the receptors PD-1 and NKG2A/C/E, suggesting a potential mechanism of tumor immune evasion. Surprisingly, therapeutic blockade of the PD-1/PD-L1 and NKG2A/Qa-1 ${ }^{\mathrm{b}}$ axes did not delay tumor growth following vaccination, suggesting that the presence of inhibitory ligands within malignant tissue may not be an effective biomarker for successful combination therapy with CMV-based vaccines. Overall, our studies show that therapeutic CMV-based vaccines in combination with adoptive $T$ cell transfer alone are effective for tumor rejection.

\section{Keywords: vaccine, checkpoint blockade, adoptive cell therapy, melanoma, cytomegalovirus}

\section{INTRODUCTION}

After decades of research, immunotherapy has finally joined surgery, radiotherapy, and chemotherapy as a standard treatment modality in clinical oncology (1-3). Currently approved immunotherapies target inhibitory receptors on T cells. Unfortunately, not all patients respond to these inhibitors. Recent work has suggested that response is correlated with T cell infiltration and an "inflamed" tumor phenotype (4). Thus, methods for converting "non-inflamed" to "inflamed" tumors are needed to treat this subset of patients. 
Vaccination is one potential method to generate an adaptive immune response against tumor cells and has been an active area of research for decades but yielding little clinical benefit as monotherapy $(5,6)$. Our lab has previously generated a novel vaccine for melanoma utilizing recombinant Cytomegalovirus (CMV) as a vaccine vector (7). Several characteristics make CMV an attractive vaccine vector: (1) CMV elicits a prolonged $\mathrm{CD} 8^{+} \mathrm{T}$ cell response characterized by the accumulation of high frequencies of virusreactive $\mathrm{T}$ cells over the lifetime of the host (8). (2) In contrast to other persistent infections, $\mathrm{CMV}$-specific $\mathrm{T}$ cells retain effector functions months after initial infection (9). (3) Prior CMV infection does not induce protective immunity, allowing for several rounds of vaccination using this vector. (4) The robust T cell response generated by $\mathrm{CMV}$ is also produced by attenuated, replication-deficient vectors (10). These properties have led several groups to test CMV vectors as vaccine platforms for infectious disease (11-13).

Our lab has previously hypothesized that a persistent vaccinestimulating lifelong $\mathrm{T}$ cell responses against tumor antigen would be an effective cancer immunotherapy $(7,14)$. To test this, our group developed a vaccine based on murine cytomegalovirus (MCMV) targeting a shared melanoma antigen, gp100. By mutating gp100 within a $\mathrm{CD} 8^{+} \mathrm{T}$ cell epitope, we were able to generate gp100-reactive $\mathrm{T}$ cells which delayed metastatic tumor progression in the lungs following vaccination with MCMVgp100KGP. However, vaccinated mice eventually succumb to disease (7). In the current study, we tested whether MCMV-based vaccines could synergize with other established immunotherapies in a murine solid tumor model of melanoma. Adoptive cell therapy is a form of immunotherapy in which large numbers of tumor-reactive $\mathrm{T}$ cells are transferred into tumor-bearing mice or humans (15). Our previous study showed that MCMVgp100KGP induced modest frequencies of tumor-reactive T cells (7). This is likely due to low precursor frequency of $\mathrm{T}$ cells recognizing the unaltered gp100 epitope. We hypothesized that adoptive cell therapy could overcome this barrier and synergize with MCMV-based vaccines to produce more effective and prolonged antitumor responses. Importantly, the effectiveness of adoptive cell therapy also seems to correlate with persistence of transferred cells long term (16). Thus, in this study, we also tested if CMV-based vaccines could sustain adoptively transferred cells long term and result in augmented tumor regression. Our results show that a MCMV-based vaccine targeting a model neo-epitope is highly effective in treating melanoma expressing the same neo-epitope, and combining this vaccination with adoptive cell therapy completely cures mice of disease. Similarly, combining MCMVgp100KGP vaccination with adoptive cell therapy delays growth of established tumors. In both models, MCMV-based vaccination maintains adoptively transferred cells at low frequencies in peripheral blood. Following vaccination, tumors take on an "inflamed" phenotype characterized by $\mathrm{T}$ cell infiltration and expression of counter-regulatory molecules, such as PD-L1 and Qa- $1^{\mathrm{b}}$. However, the protection provided by MCMV-based vaccines and adoptive T cell therapy was as effective as combination immunotherapy targeting several counter-regulatory pathways. This suggests that vaccine-induced PD-L1 and Qa-1 ${ }^{\text {b }}$ expression may not be the most accurate predictors of response to checkpoint blockade in some tumors in combination with viral-based tumor vaccines.

\section{MATERIALS AND METHODS}

\section{Mice}

Female C57BL/6 mice (6-8 weeks old) were purchased from Charles River (Frederick, MD, USA). All mice used in these studies were between 6 and 12 weeks of age at the start of the experiment. Breeding pairs of PMEL mice (B6.Cg-Thyla/ Cy $\operatorname{Tg}($ TcraTcrb)8Rest/J) were purchased from the Jackson Laboratory (Bar Harbor, ME, USA) and bred in house. OT-I/ $\mathrm{RAG}^{-/-}$mice were provided by Dr. Leo Lefrançois (University of Connecticut Health Center). Mice were housed at the University of Connecticut Health Center in a pathogen-free facility, and all experiments were performed with approval by the University of Connecticut Health Center Institutional Animal Care and Use Committee.

\section{Cell Lines and Viruses}

B16F10 and B16ova were provided by Dr. Leo Lefrançois (University of Connecticut Health Center). B16F10-RFP was purchased from AntiCancer, Inc. (San Diego, CA, USA). B16F10 cells were cultured in DMEM media (Life Technologies) supplemented with $10 \%$ fetal bovine serum (Life Technologies), $1 \mathrm{mM}$ sodium pyruvate (Life Technologies), 1\% non-essential amino acids (Life Technologies), and 1\% Penicillin/Streptomycin (Life Technologies). B16ova cells were cultured in B16F10 culture media supplemented with $500 \mu \mathrm{g} / \mathrm{mL}$ G418 (Life Technologies). B16F10-RFP were cultured according to supplier's instructions in RPMI-1640 media (Life Technologies) supplemented with 10\% fetal bovine serum, $2 \mathrm{mM}$ L-glutamine (Life Technologies), 1\% Penicillin/Streptomycin, and $400 \mu \mathrm{g} / \mathrm{mL}$ G418. Mouse embryonic fibroblasts were cultured in DMEM media supplemented with 5\% fetal bovine serum, $1 \%$ non-essential amino acids, $1 \%$ sodium pyruvate, $2 \mathrm{mM}$ L-glutamine, and 1\% Penicillin/Streptomycin. M2-10B4 (a kind gift from Dr. Christopher Snyder; Thomas Jefferson University) were cultured in RPMI-1640 media supplemented with $10 \%$ fetal bovine serum, $2 \mathrm{mM} \mathrm{L}$-glutamine, and $1 \%$ Penicillin/Streptomycin.

Murine cytomegalovirus-OVA was provided by Dr. Carol Wu (University of Connecticut Health Center). Wild-type (WT) MCMV, MCMVgp100, and MCMVgp100KGP were previously generated in the lab using the MCMV BAC pSM3fr-MCK-2fl provided by Dr. Barbara Adler (Ludwig-Maximilians-University Munich, Germany) (7). Briefly, the full-length murine gp 100 coding sequence or the altered sequence (gp100KGP) was inserted into the MCMV ie2 locus under the control of the HCMV ie1 promoter (7). Previously generated viruses (WT MCMV, MCMVgp100, and MCMVgp100KGP) were expanded using murine embryonic fibroblasts or the bone marrow stromal cell line, M2-10B4, as previously described (17).

\section{Tumor Challenge Experiments}

Female C57BL/6 mice received an intradermal injection of $10^{5}$ B16F10 or B16F10 expressing RFP (B16RFP) or $3 \times 10^{5}$ B16ova. Mice were then vaccinated via i.p. injection with $10^{5} \mathrm{PFU}$ WT or recombinant MCMV on Day 5 or Day 8, depending on the experiment. In some experiments, splenocytes corresponding 
to $10^{5} \mathrm{CD}^{+}$PMEL cells from naïve PMEL mice or $10^{5} \mathrm{CD}^{+}$ OT-I cells from OT-I/Rag ${ }^{-/-}$mice were transferred into naïve WT tumor-bearing mice $2 \mathrm{~h}$ prior to vaccination with WT or recombinant MCMV. Intradermal tumor growth was monitored every 2-3 days by measuring length and width of the tumor using calipers and multiplying to calculate surface area. Mice were euthanized when tumors reached $>100 \mathrm{~mm}^{2}$ or ulcerated. In some experiments, tumor-bearing mice also received i.p. injections of anti-PD-1 antibody (RMP1-14; BioXcell), anti-Qa-1 ${ }^{\text {b }}$ (4C2.4A7.5H11; BioXcell), or isotype controls.

\section{Flow Cytometry}

Tumor tissue was mechanically dissociated and digested in $0.7 \mathrm{mg} / \mathrm{mL}$ Collagenase D (Roche) and $3 \mathrm{mg} / \mathrm{mL}$ DNase I (Roche) for 30-45 min to obtain single-cell suspension. For experiments looking at tumor-infiltrating leukocytes (TIL), TIL were isolated using Percoll gradient prior to staining. For experiments looking at $\mathrm{B} 6 \mathrm{RFP}^{+}$tumor cells, cells were stained immediately after digestion for $20 \mathrm{~min}$. Cells were blocked with anti-CD16/32 (clone 93; Biolegend) prior to surface staining. Antibodies against the following antigens were used: CD11a (clone M17/4; ThermoFisher), CD8a (clone 53-6.7; BD Biosciences or Biolegend or eBioscience), CD45 (clone 30-F11; Invitrogen or eBioscience), CD45.1 (clone A20; Biolegend), CD3 (clone eBio500A2; eBioscience), CD45.2 (clone 104; eBioscience), CD90.1 (clone OX-7; BD Biosciences), CD127 (clone A7R34; eBioscience or clone SB/199; Biolegend), KLRG1 (clone 2F1/KLRG1; Biolegend or eBioscience), PD-1 (clone RMP1-30; eBioscience), NKG2A/C/E (clone 20d5; eBioscience), LAG3 (clone C9B7W; Biolegend), CD44 (clone IM7; eBioscience or Biolegend or BD Biosciences), Ly6C (clone HK1.4; Biolegend), Ly6G (clone 1A8; Biolegend), CD11b (M1/70; eBioscience or Biolegend), Qa-1 ${ }^{\mathrm{b}}$ (clone 6A8.6F10.1A6; Miltenyi Biotec), and PD-L1 (10F.9G2; Biolegend).

\section{Statistical Analysis}

Statistical tests were performed in Prism (Graphpad). For tumor growth experiments, tumor growth curves were compared using two-way repeated measures ANOVA. For other experiments, a student $t$-test was used when comparing two groups, and a oneway ANOVA was used when comparing more than two groups. A paired $t$-test was used to compare inhibitory receptor expression in blood and TIL from the same mouse. Survival curves were analyzed using Log-rank test in Prism.

\section{RESULTS}

\section{MCMV-OVA Maintains Adoptively Transferred Antitumor T Cells at Low Frequency Long Term}

Adoptive cell therapy is a form of cancer immunotherapy that involves infusing large numbers of ex vivo-stimulated tumorspecific T cells into patients (15). Persistence of transferred cells over time correlates with improved clinical responses following adoptive cell therapy (16). Several groups have tried to utilize the persistent nature of CMV infection to enhance this immunotherapy by redirecting CMV-specific T cells to target tumor antigen $(18,19)$. Similarly, we wondered if CMV-based vaccines could be used to enhance the persistence of adoptively transferred cells. To test this, $10^{5} \mathrm{OT}-\mathrm{I} \mathrm{CD}^{+} \mathrm{T}$ cells were transferred into naive mice that were then vaccinated with either $10^{5} \mathrm{PFU}$ WT MCMV or MCMV-OVA. As suggested by previous literature, MCMV-OVA vaccination stimulated a potent expansion of transferred T cells which was not seen following vaccination with WT MCMV (Figure 1A). Previous studies in adoptive cell therapy have shown that cells with a memory phenotype persist longer in recipients (20). We, therefore, assessed the phenotype of transferred OT-I cells following MCMV-OVA vaccination. Similar to previous studies, stimulated OT-I cells displayed an "effector memory" phenotype $\left(\mathrm{KLRG}^{+} \mathrm{CD} 127^{\mathrm{lo}}\right)$ in peripheral blood suggestive of a short-lived cell population (Figure 1B). Despite this same phenotype, MCMV inflationary $\mathrm{T}$ cell populations continue to accumulate over time due to continuous expansion of a small number of memory cells $(9,21)$. We, therefore, asked if transferred OT-I cells stimulated by MCMV-OVA persisted long term. To test this, mice were treated as in Figure 1A and OT-I frequency was followed over time in peripheral blood of mice. The majority of mice showed a progressive decline in OT-I frequency over time. Four months after transfer, OT-I cells were still detectable $\left(<2 \%\right.$ of $\left.\mathrm{CD}^{+}\right)$in blood in the majority of mice tested, and 1 of 10 mice showed a significant frequency of OT-I cells $\left(8 \%\right.$ of $\left.\mathrm{CD}^{+}\right) 4$ months after transfer (Figure 1C). While OT-I cells were still detectable in the majority of vaccinated mice several months after transfer, the frequency of transferred cells is much lower than we had hypothesized or had been shown in a previous study using recombinant MCMV (22).

\section{Adoptive Cell Transfer Enhances Efficacy of MCMV-OVA in Treatment of B16ova}

Despite the low frequency of transferred cells at late time points, we were still curious if combination adoptive cell therapy and MCMV vaccination could impact solid tumor growth of a highly aggressive melanoma tumor. To test if adoptive cell therapy and MCMV-OVA could control disease in a solid tumor model, mice were inoculated with $3 \times 10^{5} \mathrm{~B} 16$ ova on the rear flank. Eight days later when tumors were visible, mice were injected with $10^{5} \mathrm{OT}-\mathrm{I}$ cells followed by $10^{5}$ PFU WT MCMV or MCMV-OVA. As seen in our previous study, mice receiving MCMV-OVA alone showed a delay in tumor growth and prolonged survival, yet ultimately succumbed to disease. By contrast, three of five mice receiving OT-I transfer and MCMV-OVA vaccination completely cleared their tumors and remained tumor-free for the remainder of the experiment (Figure 2). Thus, despite the low persistence of transferred cells in vaccinated mice, combination adoptive cell therapy and recombinant MCMV vaccination were able to cure mice of tumors expressing a foreign antigen.

\section{MCMVgp100KGP Stimulates Adoptively Transferred PMEL Cells but Maintains Them at Low Frequency Long Term}

We next sought to determine how a CMV-based vaccine targeting an endogenous tumor antigen, gp100, would maintain antitumor $\mathrm{T}$ cell responses. Our lab has previously developed a 

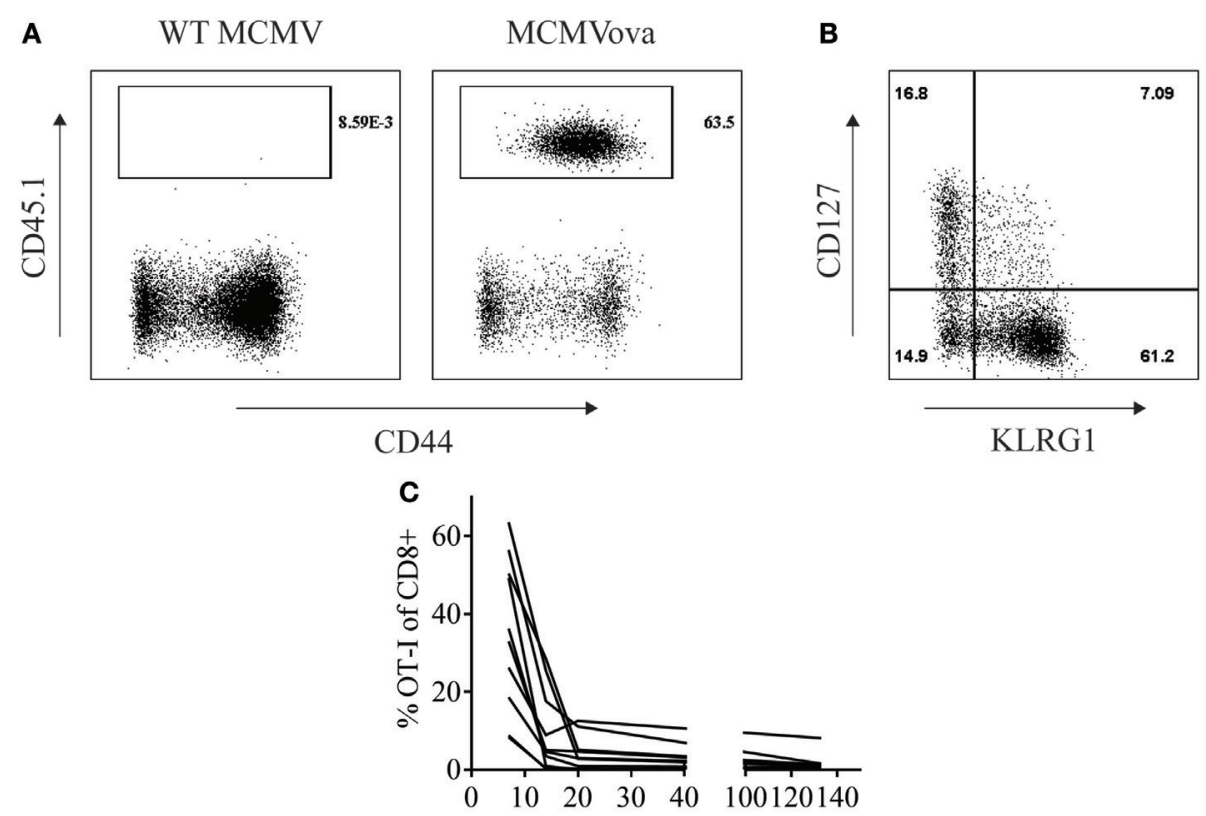

KLRG1

Days Post Transfer

FIGURE 1 | Murine cytomegalovirus (MCMV)-OVA maintains adoptively transferred OT-I cells at low frequency long-term. Wild-type (WT) CD45.2+ mice received $10^{5}$ CD45.1+ OT-I cells i.v. followed by i.p. vaccination with $10^{5}$ WT MCMV or MCMV-OVA. Peripheral blood was collected at various time points after transfer and processed for flow cytometry. (A) Representative plots from peripheral blood of mice receiving OT-I transfer and vaccination with WT MCMV or MCMV-OVA 7 days prior. Gated on $\mathrm{CD} 8^{+}$cells. (B) Representative plot showing phenotype of OT-I cells from mice vaccinated with MCMV-OVA 7 days prior. Gated on CD8 $8^{+} \mathrm{CD} 45.1^{+}$ cells. (C) Frequency of CD45.1+ of CD8 ${ }^{+}$cells over time in peripheral blood of mice vaccinated with MCMV-OVA. Each curve is representative of one mouse. Data are accumulated from two independent experiments with 10 mice/group total.
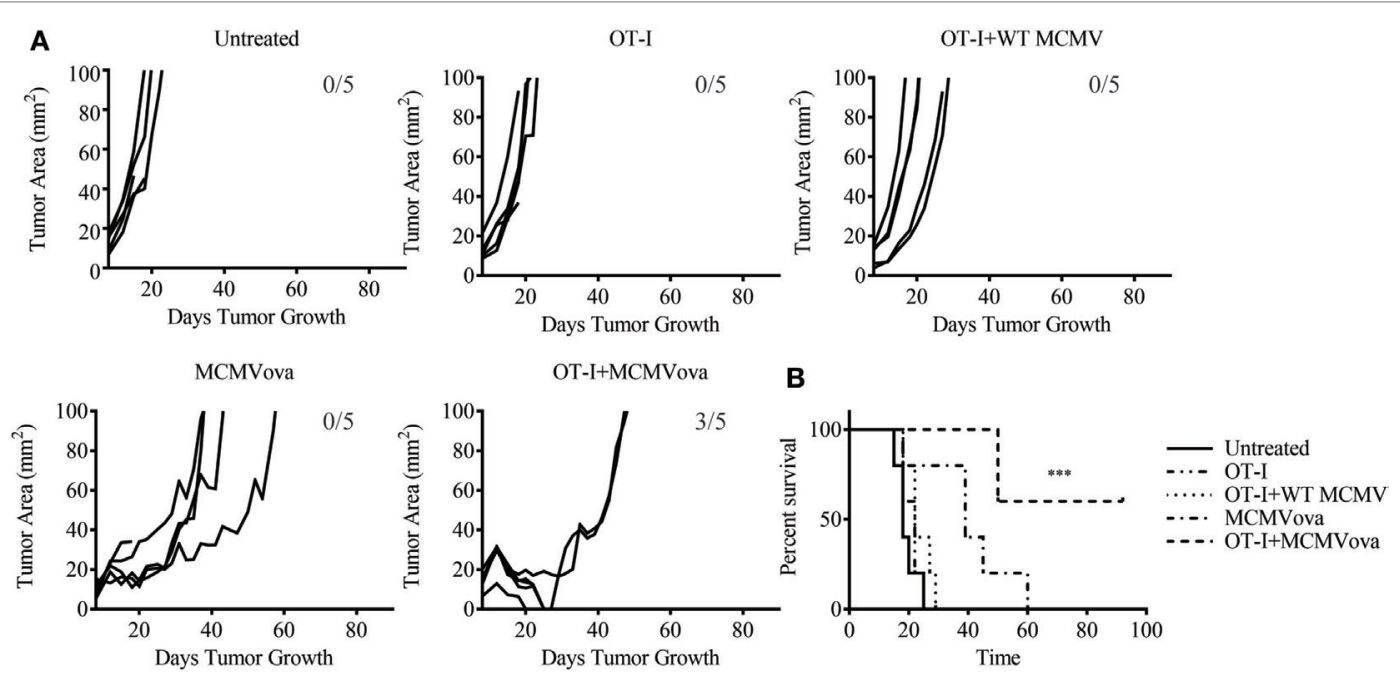

FIGURE 2 | Adoptive cell therapy and murine cytomegalovirus (MCMV)-OVA vaccination cures mice of B16ova. Wild-type (WT) mice were challenged with B16ova i.d. and 8 days later were left untreated, treated with $10^{5}$ OT-I cells i.v., treated with $10^{5}$ PFU MCMV-OVA i.p., treated with OT-I transfer i.V. and $10^{5}$ PFU WT MCMV i.p., or treated with OT-I transfer i.v. and MCMV-OVA i.p. (A) Tumor growth curves of individual mice treated as shown. (B) Survival curves for experiment shown in (A). $n=5$ mice. Number of tumor-free mice at end of experiment shown in (A). Data are representative of three independent experiments. ${ }^{\star \star \star} p<0.001$.

recombinant MCMV expressing the modified melanoma antigen, gp100KGP (7). This virus expresses murine gp100 containing a mutation within a known $\mathrm{CD}^{+} \mathrm{T}$ cell epitope, which generates an endogenous $\mathrm{T}$ cell response to the native peptide. We first wanted to determine if MCMVgp100KGP could stimulate $\mathrm{CD}^{+}$PMEL cells which express a transgenic $\mathrm{T}$ cell receptor that 
recognizes the same epitope within gp100. To test this, naïve WT mice were injected with $10^{5} \mathrm{CD}^{+}$PMEL cells followed by vaccination with WT MCMV, MCMV expressing native gp100, or MCMVgp100KGP. Five days following transfer, PMEL cells were detected at high frequency only in the blood of mice vaccinated with MCMVgp100KGP (Figure 3A), while recombinant virus expressing native gp100 did not stimulate transferred PMEL cells. We next asked if MCMVgp100KGP could promote the persistence of adoptively transferred PMEL cells. Similar to our findings with MCMV-OVA, MCMVgp100KGP stimulated a large expansion of adoptive transferred cells early but did not maintain transferred cells at high frequency in the blood long term (Figure 3B). Thus, in two models, MCMV-based vaccines maintain adoptively transferred cells in peripheral blood at low frequency long-term.

\section{Adoptive Cell Therapy and MCMVgp100KGP Vaccination Delays Solid Tumor Growth Therapeutically}

Given the encouraging results seen with adoptive transfer and MCMV-OVA vaccination, we next asked if this same combination therapy could treat tumors when targeting a native tumor antigen. To test this, mice were inoculated with $10^{5} \mathrm{~B} 16 \mathrm{~F} 10$, and 5 days later, mice received $10^{5} \mathrm{CD}^{+}$PMEL cells followed by vaccination with WT MCMV or MCMVgp100KGP. As expected, mock vaccinated tumors grew unopposed with most mice succumbing to disease within 2 weeks (Figure 4). PMEL transfer alone, MCMVgp100KGP vaccination alone, or PMEL transfer followed by WT MCMV vaccination also had little effect on tumor growth. However, PMEL transfer followed by MCMVgp100KGP vaccination significantly delayed tumor growth and prolonged survival of tumor-bearing mice. This shows that increasing the precursor frequency of antitumor $\mathrm{T}$ cells through adoptive transfer can greatly enhance the clinical efficacy of MCMV-based vaccines in an antigen-specific manner.

\section{MCMVgp100KGP-Stimulated T Cells Express Inhibitory Receptors in the Tumor Microenvironment and Induce Expression of Immunosuppressive Pathways in} Tumors

We next asked why our combination therapy did not completely clear tumors. To this end, we analyzed the phenotype of TIL for clues. Six days following vaccination with MCMVgp100KGP, PMEL cells within the tumor microenvironment expressed high levels of the inhibitory receptors LAG3, PD-1, and NKG2A compared to PMEL cells in the blood (Figure 5). Previous studies have suggested that expression of multiple inhibitory receptors correlates with cell exhaustion (23). PD-1 has two known ligands, PD-L1 and PD-L2, whose expression are regulated by IFN $\gamma$. NKG2A is an inhibitory receptor found on activated NK cells and some $\mathrm{CD}^{+} \mathrm{T}$ cells, and it recognizes the murine homolog of HLA-E, Qa- $1^{\mathrm{b}}$, whose expression is also regulated by IFN $\gamma$. Given the high expression of these inhibitory receptors on the surface of $\mathrm{T}$ cells within the tumor tissue, we next asked if the corresponding ligands were expressed within the tumor microenvironment. To test this, mice were inoculated with $10^{5} \mathrm{~B} 16 \mathrm{RFP}$ and 5 days later were left untreated or given PMEL cells followed by vaccination with WT MCMV or MCMVgp100KGP. Six days following treatment, tumor tissue was excised and processed for flow cytometry. B16RFP cells recovered from untreated or control treated tumors showed little PD-L1 or Qa-1 ${ }^{\mathrm{b}}$ expression

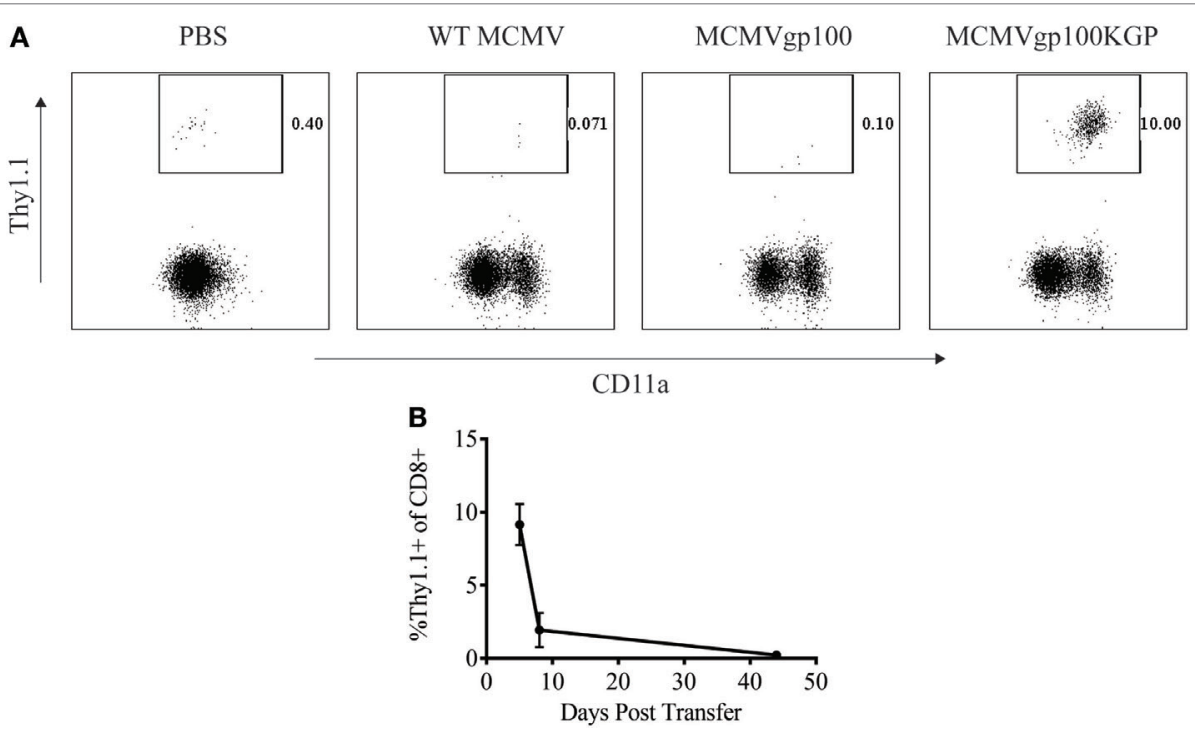

FIGURE 3 | MCMVgp100KGP maintains adoptively transferred PMEL cells at low frequency long-term. Wild-type (WT) Thy1.2+ mice received 105 Thy1.1+ PMEL cells i.v. followed by mock vaccination with PBS or vaccination with $10^{5}$ PFU WT murine cytomegalovirus (MCMV), MCMVgp100, or MCMVgp100KGP i.p. Peripheral blood was collected at various time points after transfer and processed for flow cytometry. (A) Representative plots from peripheral blood of mice receiving PMEL transfer and vaccination 5 days prior. Gated on CD8 $8^{+}$cells. (B) Frequency of Thy $1.1^{+}$of CD8 $8^{+}$cells over time in peripheral blood of mice vaccinated with MCMVgp100KGP. $n=4-5$ mice/group. Data are representative of two independent experiments. 

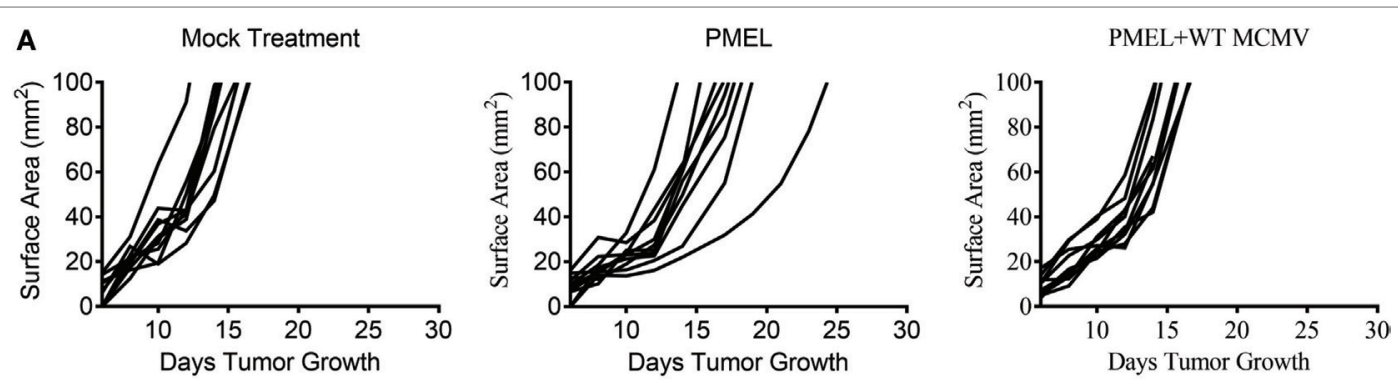

MCMVgp100KGP
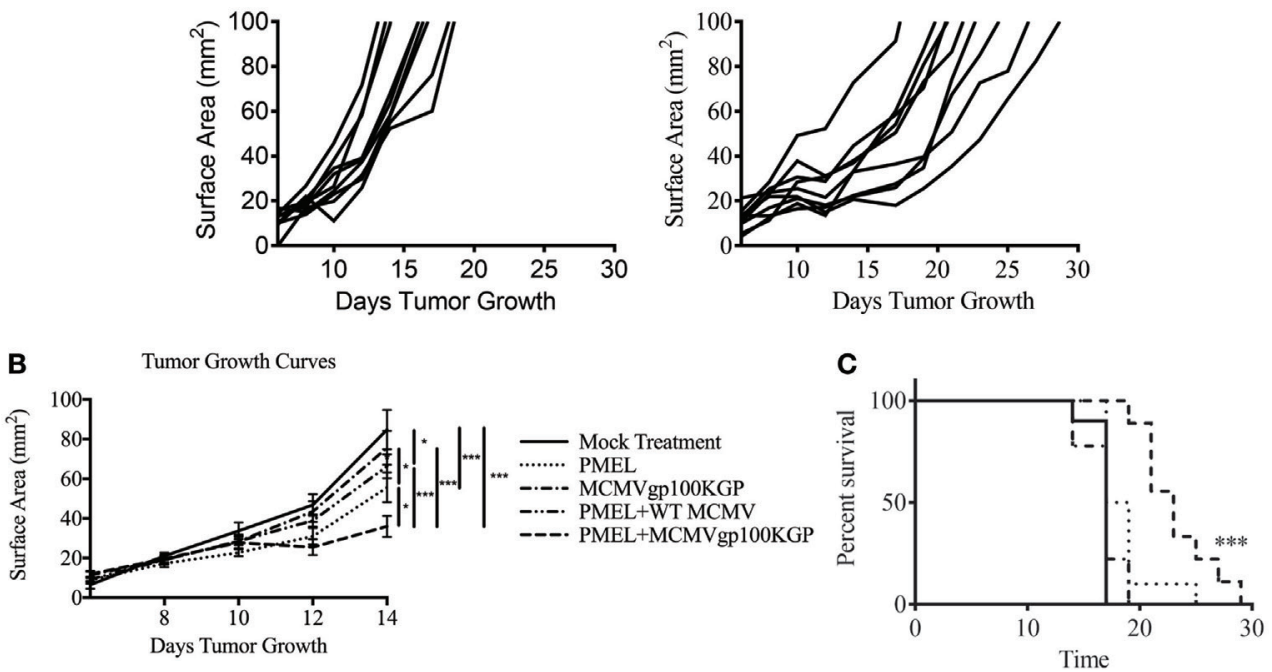

FIGURE 4 | Adoptive cell therapy and MCMVgp100KGP vaccination delays B16F10 tumor growth. Wild-type (WT) mice were challenged with B16F10 i.d. and 5 days later were mock treated, treated with PMEL transfer, treated with PMEL transfer i.v. and $10^{5}$ PFU WT MCMV i.p., treated with $10^{5}$ PFU MCMVgp100KGP i.p. alone, or treated with PMEL transfer i.v. and $10^{5}$ PFU MCMVgp100KGP i.p. (A) Tumor growth curves of individual mice treated as shown. (B) Combined tumor growth curves and (C) survival from experiment shown in (A). Data are accumulated from two independent experiments with $n=9-10$ mice/group total. One mouse from the PMEL and MCMVgp100KGP group did not grow a tumor and was excluded. ${ }^{*} p<0.05 ;{ }^{* \star *} p<0.001$.

(Figures 6A-C). Strikingly, virtually all $\mathrm{RFP}^{+}$tumor cells isolated from mice treated with MCMVgp100KGP immunotherapy were $\mathrm{PD}_{-} 1^{+}$and a significant percentage stained positive for both PD-L1 and Qa- $1^{\mathrm{b}}$ on the cell surface (Figures 6A-C). PD-L1 and Qa- $1^{\text {b }}$ were also highly upregulated on $\mathrm{CD} 45^{+} \mathrm{CD} 8^{-}$immune cells within the tumor following MCMVgp100KGP immunotherapy (Figures 6D,E). In addition to these inhibitory molecules, we also noted a substantial increase in total number of $\mathrm{Ly}_{6 \mathrm{C}} \mathrm{C}^{+}$myeloidderived suppressor cells within tumors following immunotherapy (Figure 7). These data show that immunotherapy-induced T cells rapidly induce the expression of several immunosuppressive pathways within the tumor microenvironment.

\section{PD-1 and Qa-1 ${ }^{\mathrm{b}}$ Blockade Does Not Improve Vaccination Response}

PD-1 blockade is now a well-established therapy for metastatic melanoma and other cancers, while blocking antibodies to NKG2A are currently under development for cancer therapy. Given the high expression of these inhibitory pathways following therapy, we asked whether blockade of the PD-1/PD-L1 and NKG2A/Qa-1 ${ }^{\mathrm{b}}$ axes could improve the antitumor response generated by MCMVgp100KGP vaccination. To this end, tumorbearing mice received $10^{5}$ PMEL cells and MCMVgp100KGP vaccination 5 days after tumor inoculation, followed by blocking antibodies to PD-1 and Qa- $1^{\text {b }}$ or isotype controls on days 10,12 , and 14. Surprisingly, despite the high expression of PD-1/PD-L1 and NKG2A/Qa- $1^{\mathrm{b}}$ within the tumor, blockade of these pathways had no effect on tumor growth or survival in vaccinated mice (Figure 8). This suggests that the presence of these molecules may not be effective biomarkers for response to checkpoint inhibition. Other mechanisms of resistance may also be responsible for tumor progression.

\section{DISCUSSION}

In this study, we extended our previous observations testing a novel recombinant $\mathrm{CMV}$ vaccine for melanoma. Our previous work had shown that a recombinant CMV significantly delays disease progression in a metastatic model of melanoma (7). In the current study, we sought to enhance the efficacy of vaccination by combining CMV-based vaccines with other forms of immunotherapy in a solid skin tumor model. 

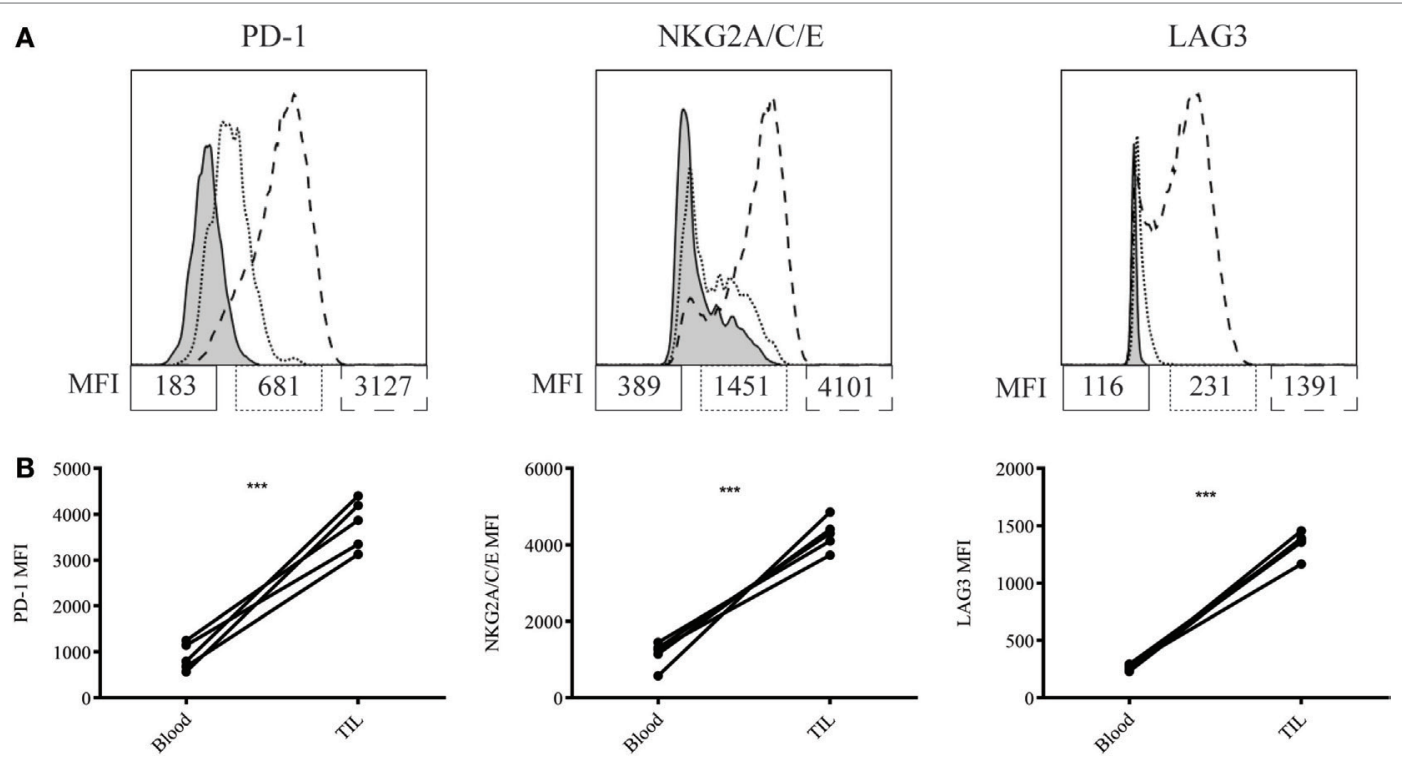

FIGURE 5 | MCMVgp100KGP stimulated PMEL cell upregulate inhibitory receptors within the tumor microenvironment. Mice bearing B16F10 tumors were treated with PMEL transfer i.v. and $10^{5}$ PFU MCMVgp100KGP vaccination i.p. Six days later, peripheral blood and tumor tissue were harvested for flow cytometry. (A) Representative plots of inhibitory receptor expression on PMEL cells from peripheral blood (dotted lines) and tumor tissue (dashed lines). Shaded curves represent FMO controls. (B) Inhibitory receptor MFI on PMEL cells from peripheral blood vs. tumor tissue. ${ }^{* \star *} p<0.001$. Paired $t$-test. $n=5$ mice/group. Data are representative of three independent experiments.

Adoptive cell therapy is a form of immunotherapy involving the transfer of high frequencies of ex vivo-stimulated antitumor $\mathrm{T}$ cells into patients (15). A prominent study in this field showed that clinical response correlated with persistence of transferred cells in patients (16). Several groups have engineered CMVspecific T cells to express receptors for tumor antigens with the hope of improving cell persistence through viral stimulation of these cells $(18,19,24)$. We, therefore, sought to ask a similar question: can a CMV-based vaccine sustain adoptively transferred antitumor T cells? Our results suggest that these cells can be maintained long-term albeit at a very low frequency. Our data stand in contrast to a recent paper by Turula et al. which showed that MCMV expressing ova could stimulate adoptively transferred OT-I cells at high frequency (22). Our conflicting results may be attributed to the different viruses used in our two studies or may be influenced by the number of cells transferred prior to vaccination. Turula et al. transfer 600 OT-I cells prior to vaccination, while in our study, mice received $10^{5}$ OT-I cells. This large frequency of transferred cells may reduce viral load and limit antigenic stimulation. Nonetheless, our data show that adoptive cell therapy greatly enhances the efficacy of CMV-based vaccines. Despite the low persistence of transferred OT-Is, OT-I transfer and MCMV-OVA vaccination completely cured mice of B16ova tumors. Similarly, PMEL transfer and MCMVgp100KGP vaccination significantly delayed growth of B16F10 tumors and increased survival of mice bearing established tumors.

Our study also highlights several immunosuppressive pathways that are upregulated in response to vaccination. PD-L1 is a notable $\mathrm{T}$ cell inhibitory ligand that is the target of several clinical immunotherapies. Clinical response to PD-1 blockade is associated with an "inflamed" tumor phenotype characterized by high levels of T cell infiltration and PD-L1 expression (25-28). B16F10 has previously been classified as a "non-immunogenic" tumor based on the resistance to immunotherapy (29). In this study, untreated B16F10 tumors express little PD-L1. Vaccination and infiltration by antitumor $\mathrm{T}$ cells significantly increases the expression of $\mathrm{PD}-\mathrm{L} 1$, suggesting that vaccination can transform B16 tumors into "inflamed" tumors. We also noted the expression of several other immunosuppressive pathways, including NKG2A and its ligand $\mathrm{Qa}-1^{\mathrm{b}}$. Qa-1 ${ }^{\mathrm{b}}$ is the murine homolog of HLA-E which has also been shown to protect malignant cells from $\mathrm{T}$ cell cytotoxicity $(30,31)$. Surprisingly, increased T cell infiltration and expression of these pathways following vaccination did not enhance the responsiveness of B16 tumors to blocking antibodies. These data highlight the difficulty in using the expression of $\mathrm{T}$ cell inhibitory molecules within the tumor microenvironment as markers for response to blocking antibodies. Instead, our data suggest that these molecules can be used as markers for successful vaccination.

Other pathways likely limit the effectiveness of vaccination in this tumor model. Herein, we also showed that vaccination induced the accumulation of $\mathrm{Ly}_{6} \mathrm{C}^{+}$myeloid cells. Given that a previous study using PMEL transfer noted a similar accumulation and showed that these cells were indeed suppressive, we hypothesize that the $\mathrm{Ly}_{6} \mathrm{C}^{+}$myeloid cells that accumulate in response to vaccination are also suppressive (32). This response may be one of the mechanisms limiting the efficacy of vaccination in our study. Future work will attempt to target these myeloid cells in combination with vaccination. 


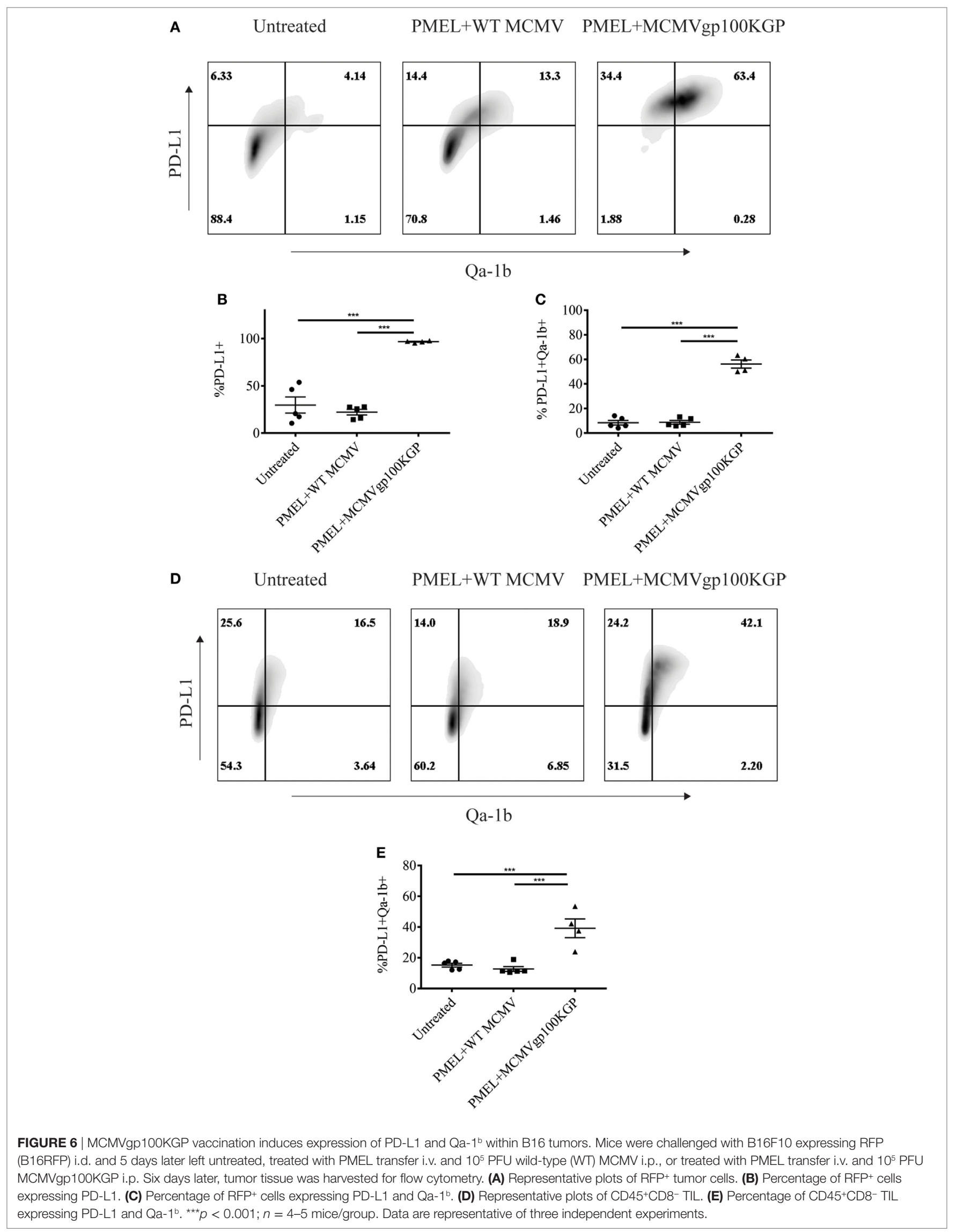



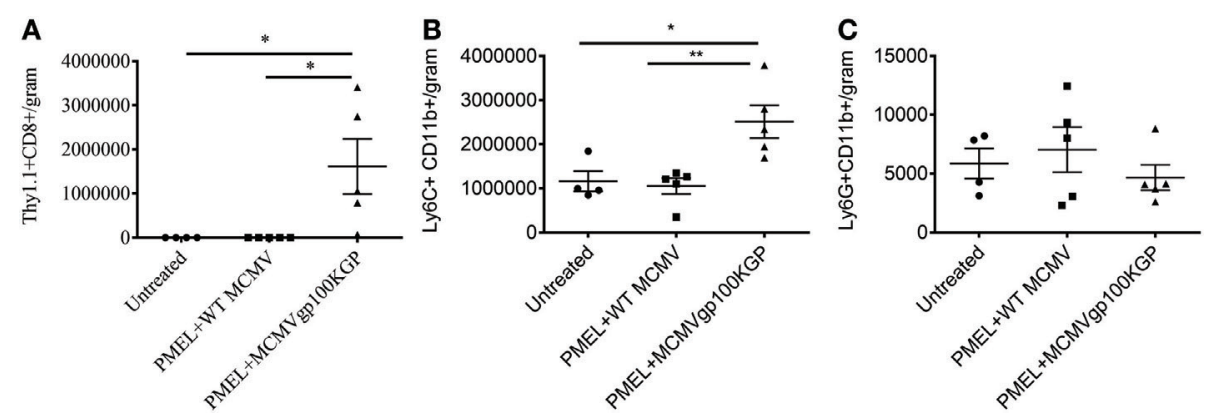

FIGURE 7 | MCMVgp100KGP vaccination induces accumulation of Ly6C+ myeloid cells. Mice bearing B16F10 tumors were treated left untreated, treated with PMEL transfer i.v. and $10^{5}$ PFU wild-type (WT) MCMV i.p., or treated with PMEL transfer i.v. and $10^{5}$ PFU MCMVgp100KGP i.p. Six days later, tumor tissue was harvested for flow cytometry. Graphs show cell counts per gram tumor tissue for (A) Thy1.1+CD8+PMEL cells, (B) Ly6C+CD11b+ cells, and (C) Ly6G+CD11b+ cells. ${ }^{\star} p<0.05 ;{ }^{* *} p<0.01 ; n=4-5$ mice/group. Data are representative of two independent experiments.
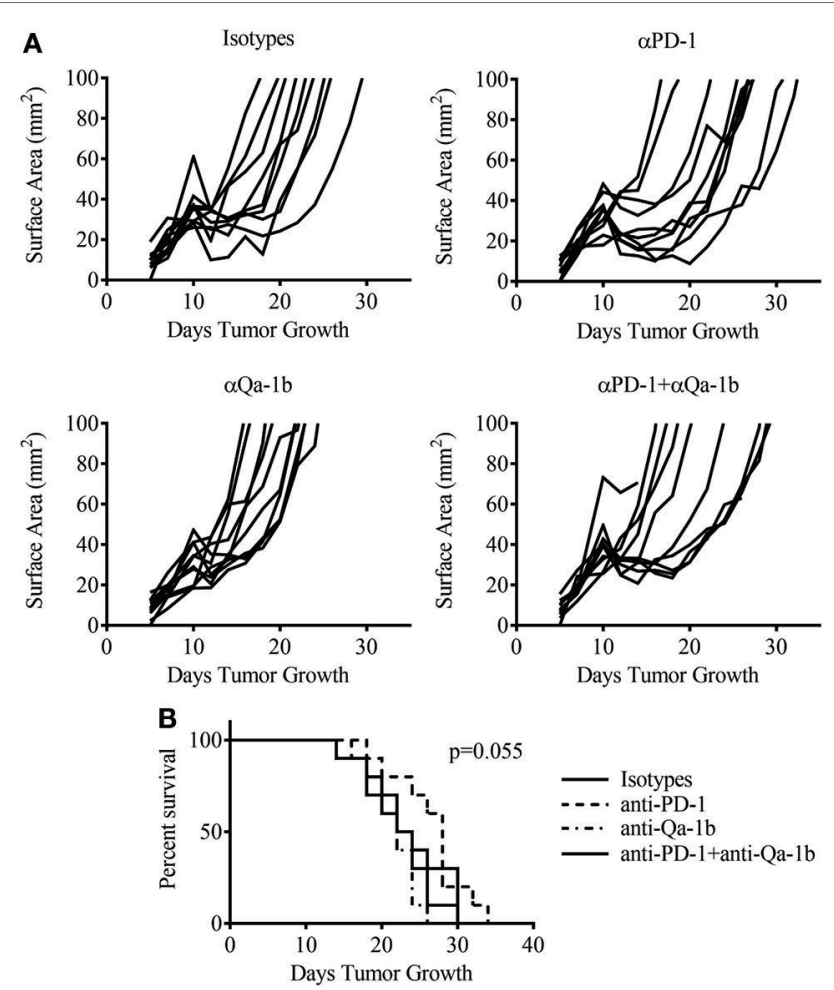

FIGURE 8 | Dual checkpoint blockade does not enhance efficacy of MCMVgp100KGP vaccination. Mice were challenged with B16F10 i.d. and 5 days later received PMEL transfer i.v. and $10^{5}$ PFU MCMVgp100KGP vaccination i.p. Mice were treated with isotype, anti-PD-1, and/or anti-Qa-1 ${ }^{\text {b }}$ antibodies i.p. on days 10,12, and 14. (A) Tumor growth curves from individual mice. (B) Survival curves from different treatment groups. Data are accumulated from two independent experiments with 10 mice/group total.

In summary, in this study, we attempted to enhance the efficacy of melanoma vaccines using CMV-based vectors. Adoptive cell therapy greatly enhanced the antitumor response to vaccination targeting both foreign and self-antigens. When targeting a foreign antigen, adoptive cell therapy and MCMV-based vaccination cured several mice of an aggressive solid tumor, suggesting that
MCMV-based vaccines against neoantigens may have dramatic clinical results. Not surprisingly, this same combination targeting a self-antigen has a less dramatic clinical response. Vaccination induced several counter-regulatory mechanisms that were not overcome by blocking antibodies, suggesting that other tumor evasion mechanisms are at play. Overall, this study extends our earlier work showing that CMV-based vaccines are effective therapies against immunosuppressive solid tumors. Future work will determine which counter-regulatory responses are limiting vaccine efficacy in this tumor model. Nevertheless, the protection provided by CMV-based vaccines is more effective than checkpoint blockade alone in the B16 model of melanoma.

\section{ETHICS STATEMENT}

The study was carried out in accordance with the University of Connecticut Health Center Institutional Animal Care and Use Committee. The protocol was approved by the University of Connecticut Health Center Institutional Animal Care and Use Committee.

\section{AUTHOR CONTRIBUTIONS}

JG and KK designed the study. JG and SY performed the experiments. ZQ and EJ provided reagents and analytic tools. JG, SY, and $\mathrm{KK}$ analyzed the data and wrote the paper.

\section{ACKNOWLEDGMENTS}

The authors would like to acknowledge Leigh Maher and Xuanmai Pham for technical assistance, Dr. Lynn Puddington and Dr. Robert Clark for helpful discussions, Dr. Leo Lefrançois, Dr. Carol Wu, and Dr. Christopher Snyder for providing reagents, and Dr. Mary Jo Turk for helpful discussion.

\section{FUNDING}

This study was funded in part by the Department of Defense grant: W81XWH-14-1-0342. 


\section{REFERENCES}

1. Hodi FS, O'Day SJ, McDermott DF, Weber RW, Sosman JA, Haanen JB, et al. Improved survival with ipilimumab in patients with metastatic melanoma. N Engl J Med (2010) 363(8):711-23. doi:10.1056/NEJMoa1003466

2. Robert C, Thomas L, Bondarenko I, O'Day S, Weber J, Garbe C, et al. Ipilimumab plus dacarbazine for previously untreated metastatic melanoma. N Engl J Med (2011) 364(26):2517-26. doi:10.1056/NEJMoa1104621

3. Robert C, Long GV, Brady B, Dutriaux C, Maio M, Mortier L, et al. Nivolumab in previously untreated melanoma without BRAF mutation. $N$ Engl J Med (2015) 372(4):320-30. doi:10.1056/NEJMoa1412082

4. Gajewski TF. The next hurdle in cancer immunotherapy: overcoming the nonT-cell-inflamed tumor microenvironment. Semin Oncol (2015) 42(4):663-71. doi:10.1053/j.seminoncol.2015.05.011

5. Melero I, Gaudernack G, Gerritsen W, Huber C, Parmiani G, Scholl S, et al. Therapeutic vaccines for cancer: an overview of clinical trials. Nat Rev Clin Oncol (2014) 11(9):509-24. doi:10.1038/nrclinonc.2014.111

6. Guo C, Manjili MH, Subjeck JR, Sarkar D, Fisher PB, Wang XY. Therapeutic cancer vaccines: past, present, and future. Adv Cancer Res (2013) 119:421-75. doi:10.1016/B978-0-12-407190-2.00007-1

7. Qiu Z, Huang H, Grenier JM, Perez OA, Smilowitz HM, Adler B, et al. Cytomegalovirus based vaccine expressing a modified tumor antigen induces potent tumor-specific CD8+ T cell response and protects mice from melanoma. Cancer Immunol Res (2015) 3(5):536-46. doi:10.1158/2326-6066. CIR-14-0044

8. Klenerman P, Oxenius A. T cell responses to cytomegalovirus. Nat Rev Immunol (2016) 16(6):367-77. doi:10.1038/nri.2016.38

9. Snyder CM, Cho KS, Bonnett EL, van Dommelen S, Shellam GR, Hill AB. Memory inflation during chronic viral infection is maintained by continuous production of short-lived, functional T cells. Immunity (2008) 29(4):650-9. doi:10.1016/j.immuni.2008.07.017

10. Snyder CM, Cho KS, Bonnett EL, Allan JE, Hill AB. Sustained CD8+ T cell memory inflation after infection with a single-cycle cytomegalovirus. PLoS Pathog (2011) 7(10):e1002295. doi:10.1371/journal.ppat.1002295

11. Beverley PC, Ruzsics Z, Hey A, Hutchings C, Boos S, Bolinger B, et al. A novel murine cytomegalovirus vaccine vector protects against Mycobacterium tuberculosis. J Immunol (2014) 193(5):2306-16. doi:10.4049/jimmunol.1302523

12. Hansen SG, Vieville C, Whizin N, Coyne-Johnson L, Siess DC, Drummond DD, et al. Effector memory $\mathrm{T}$ cell responses are associated with protection of rhesus monkeys from mucosal simian immunodeficiency virus challenge. Nat Med (2009) 15(3):293-9. doi:10.1038/nm.1935

13. Hansen SG, Ford JC, Lewis MS, Ventura AB, Hughes CM, Coyne-Johnson L, et al. Profound early control of highly pathogenic SIV by an effector memory T-cell vaccine. Nature (2011) 473(7348):523-7. doi:10.1038/nature10003

14. Qiu Z, Grenier JM, Khanna KM. Reviving virus based cancer vaccines by using cytomegalovirus vectors expressing modified tumor antigens. Oncoimmunology (2016) 5(1):e1056974. doi:10.1080/2162402X.2015.1056974

15. Rosenberg SA, Restifo NP. Adoptive cell transfer as personalized immunotherapy for human cancer. Science (2015) 348(6230):62-8. doi:10.1126/ science.aaa4967

16. Robbins PF, Dudley ME, Wunderlich J, El-Gamil M, Li YF, Zhou J, et al. Cutting edge: persistence of transferred lymphocyte clonotypes correlates with cancer regression in patients receiving cell transfer therapy. J Immunol (2004) 173(12):7125-30. doi:10.4049/jimmunol.173.12.7125

17. Zurbach KA, Moghbeli T, Snyder CM. Resolving the titer of murine cytomegalovirus by plaque assay using the M2-10B4 cell line and a low viscosity overlay. Virol J (2014) 11:71. doi:10.1186/1743-422X-11-71

18. Caruana I, Weber G, Ballard BC, Wood MS, Savoldo B, Dotti G. K562derived whole-cell vaccine enhances antitumor responses of CAR-redirected virus-specific cytotoxic T lymphocytes in vivo. Clin Cancer Res (2015) 21(13):2952-62. doi:10.1158/1078-0432.CCR-14-2998

19. Wang X, Wong CW, Urak R, Mardiros A, Budde LE, Chang WC, et al. CMVpp65 vaccine enhances the antitumor efficacy of adoptively transferred
CD19-redirected CMV-specific T cells. Clin Cancer Res (2015) 21(13):29933002. doi:10.1158/1078-0432.CCR-14-2920

20. Klebanoff CA, Gattinoni L, Palmer DC, Muranski P, Ji Y, Hinrichs CS, et al. Determinants of successful CD8+ T-cell adoptive immunotherapy for large established tumors in mice. Clin Cancer Res (2011) 17(16):5343-52. doi:10.1158/1078-0432.CCR-11-0503

21. Quinn M, Turula H, Tandon M, Deslouches B, Moghbeli T, Snyder CM. Memory T cells specific for murine cytomegalovirus re-emerge after multiple challenges and recapitulate immunity in various adoptive transfer scenarios. J Immunol (2015) 194(4):1726-36. doi:10.4049/jimmunol.1402757

22. Turula H, Smith CJ, Grey F, Zurbach KA, Snyder CM. Competition between $\mathrm{T}$ cells maintains clonal dominance during memory inflation induced by MCMV. Eur J Immunol (2013) 43(5):1252-63. doi:10.1002/eji.201242940

23. Blackburn SD, Shin H, Haining WN, Zou T, Workman CJ, Polley A, et al. Coregulation of CD8+ $\mathrm{T}$ cell exhaustion by multiple inhibitory receptors during chronic viral infection. Nat Immunol (2009) 10(1):29-37. doi:10.1038/ ni. 1679

24. Cruz CR, Micklethwaite KP, Savoldo B, Ramos CA, Lam S, Ku S, et al. Infusion of donor-derived CD19-redirected virus-specific $\mathrm{T}$ cells for B-cell malignancies relapsed after allogeneic stem cell transplant: a phase 1 study. Blood (2013) 122(17):2965-73. doi:10.1182/blood-2013-06-506741

25. Topalian SL, Hodi FS, Brahmer JR, Gettinger SN, Smith DC, McDermott DF, et al. Safety, activity, and immune correlates of anti-PD-1 antibody in cancer. N Engl J Med (2012) 366(26):2443-54. doi:10.1056/NEJMoa1200690

26. Taube JM, Anders RA, Young GD, Xu H, Sharma R, McMiller TL, et al. Colocalization of inflammatory response with B7-h1 expression in human melanocytic lesions supports an adaptive resistance mechanism of immune escape. Sci Transl Med (2012) 4(127):127ra37. doi:10.1126/ scitranslmed.3003689

27. Taube JM, Klein A, Brahmer JR, Xu H, Pan X, Kim JH, et al. Association of PD-1, PD-1 ligands, and other features of the tumor immune microenvironment with response to anti-PD-1 therapy. Clin Cancer Res (2014) 20(19):5064-74. doi:10.1158/1078-0432.CCR-13-3271

28. Tumeh PC, Harview CL, Yearley JH, Shintaku IP, Taylor EJ, Robert L, et al. PD-1 blockade induces responses by inhibiting adaptive immune resistance. Nature (2014) 515(7528):568-71. doi:10.1038/nature13954

29. Chen S, Lee LF, Fisher TS, Jessen B, Elliott M, Evering W, et al. Combination of 4-1BB agonist and PD-1 antagonist promotes antitumor effector/memory CD8 T cells in a poorly immunogenic tumor model. Cancer Immunol Res (2015) 3(2):149-60. doi:10.1158/2326-6066.CIR-14-0118

30. Gooden M, Lampen M, Jordanova ES, Leffers N, Trimbos JB, van der Burg SH, et al. HLA-E expression by gynecological cancers restrains tumor-infiltrating CD8(+) T lymphocytes. Proc Natl Acad Sci U S A (2011) 108(26):10656-61. doi:10.1073/pnas.1100354108

31. Malmberg KJ, Levitsky V, Norell H, de Matos CT, Carlsten M, Schedvins K, et al. IFN-gamma protects short-term ovarian carcinoma cell lines from CTL lysis via a CD94/NKG2A-dependent mechanism. J Clin Invest (2002) 110(10):1515-23. doi:10.1172/JCI0215564

32. Hosoi A, Matsushita H, Shimizu K, Fujii S, Ueha S, Abe J, et al. Adoptive cytotoxic $\mathrm{T}$ lymphocyte therapy triggers a counter-regulatory immunosuppressive mechanism via recruitment of myeloid-derived suppressor cells. Int J Cancer (2014) 134(8):1810-22. doi:10.1002/ijc.28506

Conflict of Interest Statement: The authors declare that the research was conducted in the absence of any commercial or financial relationships that could be construed as a potential conflict of interest.

Copyright (๑) 2018 Grenier, Yeung, Qiu, Jellison and Khanna. This is an open-access article distributed under the terms of the Creative Commons Attribution License (CC BY). The use, distribution or reproduction in other forums is permitted, provided the original author(s) or licensor are credited and that the original publication in this journal is cited, in accordance with accepted academic practice. No use, distribution or reproduction is permitted which does not comply with these terms. 